\title{
Biopolymer Skeleton Produced by Rhizobium radiobacter: Stoichiometric Alternation of Glycosidic and Amidic Bonds in the Lipopolysaccharide O-Antigen
}

Speciale, Immacolata; Di Lorenzo, Flaviana; Gargiulo, Valentina; Erbs, Gitte; Newman, Mari Anne; Molinaro, Antonio; De Castro, Cristina

\section{Published in:}

Angewandte Chemie - International Edition

Link to article, DOI:

10.1002/anie.201914053

10.1002/ange.201914053

Publication date:

2020

Document Version

Peer reviewed version

Link back to DTU Orbit

Citation (APA):

Speciale, I., Di Lorenzo, F., Gargiulo, V., Erbs, G., Newman, M. A., Molinaro, A., \& De Castro, C. (2020). Biopolymer Skeleton Produced by Rhizobium radiobacter: Stoichiometric Alternation of Glycosidic and Amidic Bonds in the Lipopolysaccharide O-Antigen. Angewandte Chemie - International Edition, 59(16), 6368-6374. https://doi.org/10.1002/anie.201914053, https://doi.org/10.1002/ange.201914053

\section{General rights}

Copyright and moral rights for the publications made accessible in the public portal are retained by the authors and/or other copyright owners and it is a condition of accessing publications that users recognise and abide by the legal requirements associated with these rights.

- Users may download and print one copy of any publication from the public portal for the purpose of private study or research.

- You may not further distribute the material or use it for any profit-making activity or commercial gain

- You may freely distribute the URL identifying the publication in the public portal 


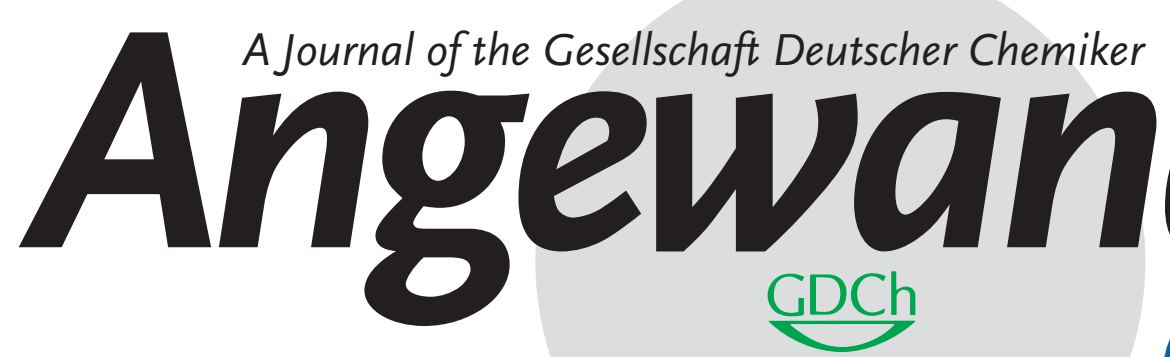

International Edition

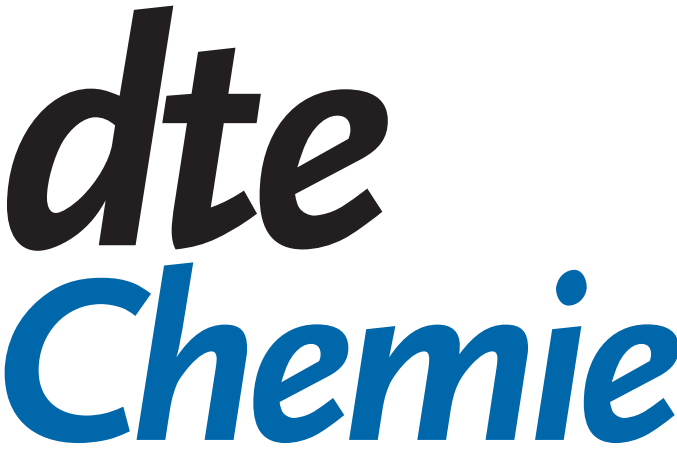

www.angewandte.org

\section{Accepted Article}

Title: A new biopolymer skeleton is produced by Rhizobium radiobacter: stoichiometric alternation of glycosydic and amidic bonds in the LPS O-Antigen

Authors: Immacolata Speciale, Flaviana Di Lorenzo, Valentina Gargiulo, Gitte Erbs, Mari-Anne Newman, Antonio Molinaro, and Cristina De Castro

This manuscript has been accepted after peer review and appears as an Accepted Article online prior to editing, proofing, and formal publication of the final Version of Record (VoR). This work is currently citable by using the Digital Object Identifier (DOI) given below. The VoR will be published online in Early View as soon as possible and may be different to this Accepted Article as a result of editing. Readers should obtain the VoR from the journal website shown below when it is published to ensure accuracy of information. The authors are responsible for the content of this Accepted Article.

To be cited as: Angew. Chem. Int. Ed. 10.1002/anie.201914053

Angew. Chem. 10.1002/ange.201914053

Link to VoR: http://dx.doi.org/10.1002/anie.201914053

http://dx.doi.org/10.1002/ange.201914053 


\title{
A New Biopolymer Skeleton Produced by Rhizobium radiobacter: Stoichiometric Alternation of Glycosidic and Amidic bonds in the Lipopolysaccharide O-Antigen
}

\author{
Immacolata Speciale, ${ }^{[a] \S}$ Flaviana Di Lorenzo, ${ }^{[a] \S}$ Valentina Gargiulo, ${ }^{[a, b]}$ Gitte Erbs, ${ }^{[c]}$ Mari-Anne Newman, ${ }^{[d]}$
} Antonio Molinaro, ${ }^{*[a]}$ Cristina De Castro*[e]

\begin{abstract}
The lipopolysaccharide (LPS) O-antigen structure of the plant pathogen Rhizobium radiobacter strain TT9 and its possible role in a plant-microbe interaction was investigated. The analyses disclosed the presence of two O-antigens, named Poly1 and Poly2. The repetitive unit of Poly2 is constituted by $4-\alpha-L-$-rhamnose linked to a $3-\alpha-D-f u c o s e$ residue. Surprisingly, Poly1 turned out to be a novel type of biopolymer in which the repeating unit is formed by a monosaccharide and an amino acid derivative, so that the polymer has alternating glycosidic and amidic bonds joining the two units: 4-amino-4-deoxy-3-O-methylD-fucose and $\left(2^{\prime} R, 3^{\prime} R, 4^{\prime} S\right)-N$-methyl-3',4'-dihydroxy-3'-methyl-5'oxoproline).

Differently from the O-antigens of LPSs from other pathogenic Gram-negative bacteria, these two O-antigens do not activate the oxidative burst, an early innate immune response in the model plant Arabidopsis thaliana, explaining at least in part the ability of this $R$. radiobacter strain to avoid host defenses during a plant infection process.
\end{abstract}

\section{Introduction}

Microbial surface antigens are key molecules for both symbiotic and pathogenic bacteria involved in the interaction with other organisms and/or with the external environment. They are mostly carbohydrate-based molecules such as glycolipids, glycoproteins and (capsular) polysaccharides. In Gram-negative bacteria, the LPS represents the most important and abundant membrane/surface antigen. ${ }^{[1-3]}$ LPSs play a key role in the elicitation of innate immune response in all eukaryotes and are recognized as MAMPs (Microbe Associated Molecular Patterns) by the host immune system. ${ }^{[4]}$

\footnotetext{
[a] Prof. A. Molinaro, Dr. F. Di Lorenzo, Dr. I. Speciale, Dr. V. Gargiulo Department of Chemical Sciences, University of Napoli Via Cintia 4, 80126 Napoli (Italy) E-mail: molinaro@unina.it

[b] Dr. V. Gargiulo. Institute for Research on Combustion (IRC), CNR, Naples, Italy Piazzale Vincenzo Tecchio 80, 80126 Napoli, Italy

[c] Dr. G. Erbs

Department of Health Technology, Technical University of Denmark, Kemitorvet, 2800 Kgs. Lyngby, Denmark

[d] Prof. M.-A. Newman Department of Plant and Enviromental Sciences, Faculty of Science, University of Copenhagen, Thorvaldsensvej 40, 1871 Frederiksberg Denmark.

[e] Prof. C. De Castro Department of Agricultural Sciences, University of Napoli Via Università 100, 80055 Portici (NA) (Italy) E-mail: decastro@unina.it
}

\section{§ Equal contribution}

Supporting information (including extraction methods, chemical and spectroscopical procedures, computational details, data discussion and NMR interpretation) of this article can be found under: https://.
LPS comprises three chemically and genetically distinct regions: the endotoxic moiety termed Lipid $A$, the core region and the O-specific polysaccharide (OPS) or O-antigen, ${ }^{[1-3]}$ that is the predominant part by weight of the LPS.

We and several other groups have demonstrated that in the course of a symbiotic interaction, the bacterial LPS O-antigens play a crucial role in the suppression of the plant innate immunity by mimicking host surface carbohydrate antigens, ${ }^{[5]}$ or that they circumvent recognition by building up a chemically unique and tricky repeating unit endowed with helicoidal supramolecular structures. $^{[6]}$ On the contrary, when the bacterium establishes pathogenic interactions, the OPS is able to elicit plant defense responses. ${ }^{[7,8]}$

In the frame of our ongoing investigation and with the aim to evaluate the role of the LPS O-antigen in a plant interaction with a pathogenic strain of Rhizobium, we studied Rhizobium radiobacter strain TT9 (here named RrTT9; previously named Agrobacterium tumefaciens) ${ }_{1}^{[9]}$ the causative agent of the crown gall disease.

\section{Results and Discussion}

Isolation of the 5-oxoproline derivative of the methyl glycoside of fucosamine (1 $\alpha$ and $1 \beta)$. Briefly (full discussion in Supporting Information), bacterial cells extraction by the hot water/phenol method afforded LPS mainly in the phenol phase and the compositional analysis by GLC-MS detected three main components: 4-substituted L-rhamnose (L-Rha), 3-substituted Dfucose (D-Fuc), whereas the nature of third one was understood only after the NMR study of the isolated compound. Indeed, RrTT9 LPS underwent solvolysis with $2 \mathrm{M} \mathrm{MeOH} / \mathrm{HCl}$, and $\mathrm{HPLC}$ purification yielded to the $\alpha / \beta$ mixture of 1 (Figure 1).

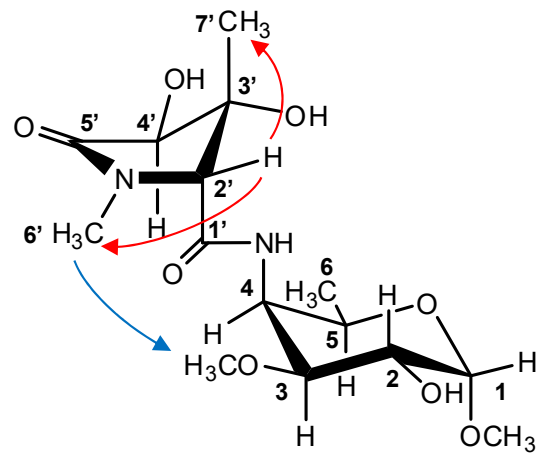

Figure 1. Structure of the O-methyl- $\alpha$-glycoside $(1 \alpha)$ of the 4-amino-4-deoxy-3O-methyl-D-fucose amidated at N-4 by the $\left(2^{\prime} R, 3^{\prime} R, 4 ' S\right) N$-methyl-3',4'dihydroxy-3'-methyl-5'-oxoproline. Arrows indicate the intra- (red) and inter(blue) residue NOE effects relevant to establish the stereochemistry of the aglycon moiety. 
Identification of 1 as a methyl-4-amino-4-deoxy-3-O-methylfucoside with an oxoproline derivative $\left(\left(2^{\prime} R, 3^{\prime} R, 4^{\prime} S\right)-N\right.$-methyl3',4'-dihydroxy-3'-methyl-5'-oxoproline) in amide linkage at position 4, was possible by combining NMR and GLC-MS studies.

The HSQC spectrum of the mixture of $1 \alpha$ and $1 \beta$ (Figure S1, Table S1) showed two anomeric densities at 4.82/100.6 and $4.35 / 105.5 \mathrm{ppm}\left({ }^{1} \mathrm{H} /{ }^{13} \mathrm{C}\right)$; both anomeric signals gave evidence of a 6-deoxy-galacto-configured residue on the basis of the small values of the ${ }^{3} J_{\mathrm{H} 3, \mathrm{H} 4}(4.3 \mathrm{~Hz}$ for $1 \mathbf{a}$ and $4.6 \mathrm{~Hz}$ for $1 \mathbf{b})$ and ${ }^{3} J_{\mathrm{H} 4, \mathrm{H} 5}$ $(1.5 \mathrm{~Hz}$, same for $\mathbf{1 a}$ and $\mathbf{1 b})$. Further, HSQC spectrum correlated $\mathrm{H}-4$ proton signal to a carbon at $51.6 \mathrm{ppm}$, a value indicative of a nitrogen atom (either aminic or amidic) attached, whereas the low field C-3 values (79.2 and $82.5 \mathrm{ppm}$ in $\alpha / \beta$ anomers, respectively) together with the presence of a methoxyl signal at 3.38/58.2 ppm indicated this last group was linked at $\mathrm{C}-3$, as confirmed by the corresponding correlation in the HMBC spectrum (Fig. S1). Therefore, the merge of these data indicated the presence of the O-methyl glycoside of a 4-amino-4-deoxy-3-O-methyl-fucose (3OMeFuc4N).

However, the HSQC spectrum contained additional crosspeaks, which fitted with the skeleton of a 5-oxoproline ring (Figure 1, labelled as $\mathbf{A g}$ ), substituted with two hydroxyl functions at C-3' and $\mathrm{C}-4$ ', and with two methyl groups, one at C-3' and the other $\mathrm{N}$ linked to the lactam nitrogen, as inferred by analysis of the HMBC spectrum (Figure S1, Supporting Information for discussion).

Information about the relative stereochemistry of the stereocenters at C-2', C-3' and C-4', was acquired by analyzing the ROESY spectrum (Figure S2). Briefly, the proton signal at C-2' $\left(\mathbf{A g}_{2}\right)$ showed NOE contacts (red arrows in Figure 1) with the methyl protons of both C-6' $\left(\mathbf{A g}_{6^{\prime}}\right)$ and C-7' $\left(\mathbf{A g}_{7^{\prime}}\right)$, suggesting their occurrence on the same side of the oxoproline ring. On the contrary, none of these protons showed a NOE correlation with the hydroxymethine proton at $\mathrm{C}^{-4^{\prime}}\left(\mathbf{A g}_{4^{\prime}}\right)$. Finally, the inter-residue NOE contact (blue arrows in Figure 1) between the $N$-methyl protons $\left(\mathbf{A g}_{6^{\prime}}\right)$ and the $\mathrm{O}$-methyl proton signal at $\mathrm{C}-3$ of the sugar unit $\left(\mathbf{A}_{\mathbf{3}} \mathrm{OMe}\right)$ indicated that these two units were linked together (Figure S2). Hence, based on the NOEs observed, it was possible to define the relative orientation of all the substituents, consistent with the stereochemistry $S, S$ and $R$ for the stereocenters C-2', C$3^{\prime}$ and $C-4^{\prime}$ or with their mirror image $\left(2^{\prime} R, 3^{\prime} R, 4^{\prime} S\right)$. The oxoproline group was linked as amide to the amino function of the $30 \mathrm{Me}-$ Fuc4N unit as suggested by downfield displacement of $\mathrm{H}-4$ chemical shift due to acylation (Table S1).

The information acquired by NMR for 1 , enabled the understanding of the unassigned peak in GLC-MS mass spectrum (Figure 2).

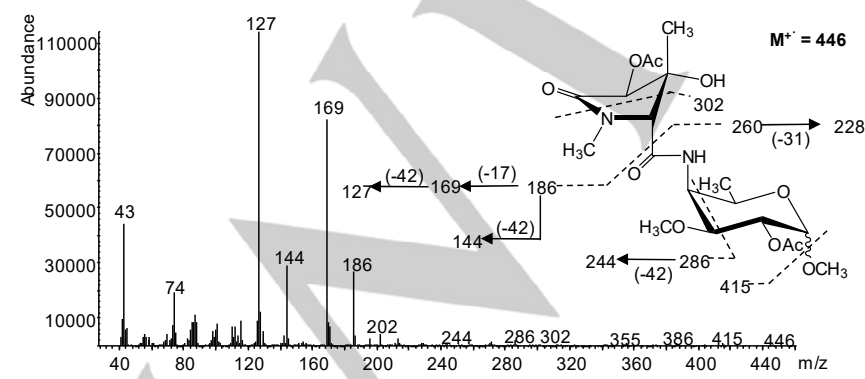

Figure 2. Mass spectrum of the acetylated methyl glycoside derivative. Interpretation of the main fragments is reported in the inset.

The molecular ion at $\mathrm{m} / \mathrm{z} 446$ (Figure 2) matched the value expected for the acetylated methyl glycoside, and the fragment at $\mathrm{m} / \mathrm{z} 186$ was consistent with the fragment of the aglycon without the sugar portion, which in turn generated the secondary fragments by loss of $\mathrm{OH}(17 \mathrm{u})$ and ketene $(42 \mathrm{u})$. The structure of such derivative and of its key fragments were further confirmed by analyzing the fully methylated form of the same methyl glycoside (Figure S3).

Smith degradation of the LPS fraction, and characterization of Poly1. Next, linkage analysis on the intact LPS showed 3-D-Fuc and 4-L-Rha but no derivative of 2 -linked $30 M$ MeFuc4N, suggesting that 1 was inserted in the OPS chain by involving one of the hydroxyl functions of the aglycon. In order to disclose the structure of the OPS, Smith degradation was performed to degrade 4-L-Rha, the only unit sensitive to this procedure (see Supporting Information). Surprisingly, the NMR analysis (Table S2, Figures 4 and S4) of the reaction product displayed that it was a homopolymer, named Poly1 (Figure 3), made by $\mathbf{1}$ (labelled as $\mathbf{C}$ in the spectra) in the $\alpha$ configuration at the anomeric centre, as deduced from the $J_{\mathrm{H} 1, \mathrm{H} 2}$ value of $2.9 \mathrm{~Hz}$.

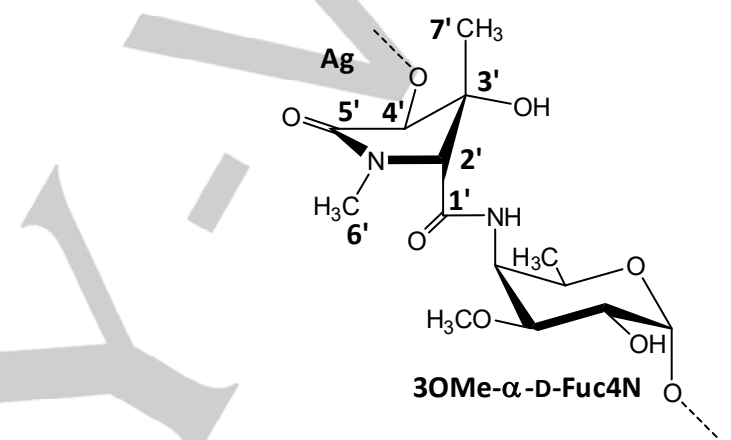

Figure 3. Structure of Poly1 O-antigen produced by RrTT9: a new biopolymer composed of 4-amino-4-deoxy-3-O-methyl-D-fucose amidated at $\mathrm{N}-4$ with $(2$ ' $R$, 3'R, 4'S) N-methyl-3,4-dihydroxy-3-methyl-5-oxoproline abbreviated as Ag.

Importantly, the HSQC spectrum (Figure 4) disclosed that glycosylation occurred at O-4' of the aglycon based on the shift at lowfield of the corresponding carbon $(80.0 \mathrm{ppm})$ compared to the value found for the methyl glycoside (76.6 ppm, Table S1), as anticipated from the results of linkage analysis.

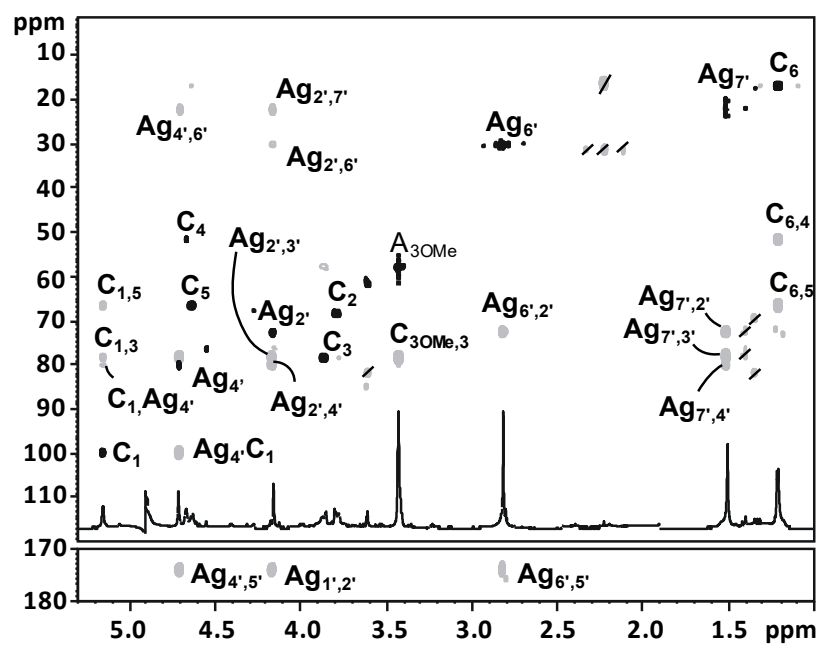

Figure 4. HSQC (black) and HMBC (grey) spectra of the Poly1 repeating unit (600 MHz, $290 \mathrm{~K}, \mathrm{D}_{2} \mathrm{O}$; Table S2). "l" symbol indicates impurities. 


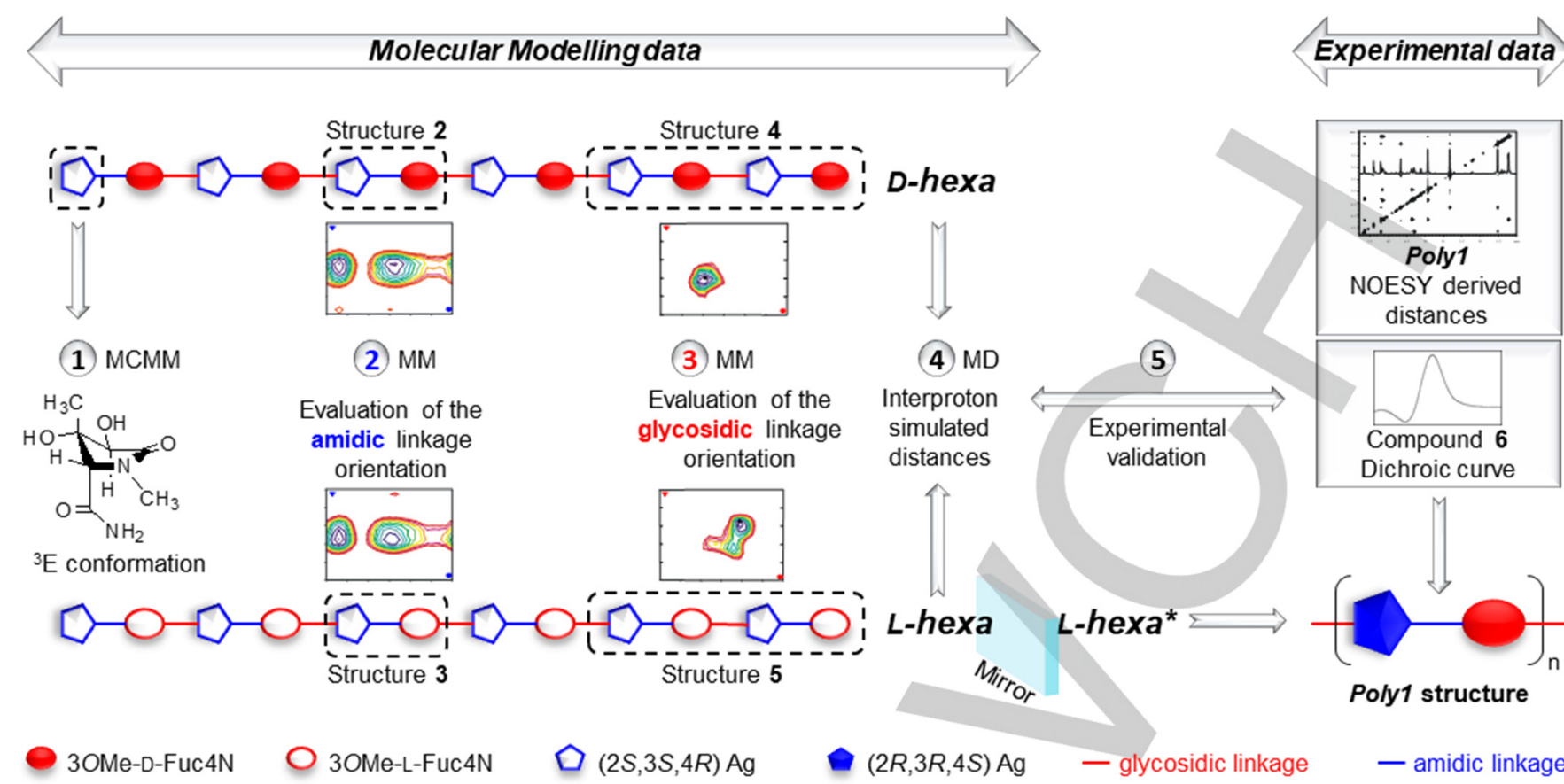

Scheme 1. Representation of the strategy used to evaluate the absolute stereochemistry of Poly1 and of its monomer, 1 (Figure 1 ). For simplicity, the D- and Lhexa oligomers (Figure 5) have been drawn as a cartoon, and the whole strategy has been dissected into five steps. The Molecular Modelling data section includes four steps, each performed on a specific structural motif. In steps 1-3, this motif is circled within the hexamer with a dotted box, while in step 4 , the molecule examined is the hexamer proper. The region between the two hexamers reports the kind of investigation done, and sketches the main results obtained. As for the experimental section, the data used for validation of the modeling data (step 5) are derived from NMR (NOESY spectrum derived distances) and Circular Dichroism measurements. The precise structures of 2-5 and of L- and D-hexa are reported in Figure 5. MCMM: Monte Carlo Multiple minimum conformational search, MM: Molecular Mechanic; MD: Molecular Dynamic.

The information from the HSQC was confirmed by analyzing the HMBC spectrum (Figure 4) which contained two key correlations, $\mathbf{A g}_{4} \cdot \mathbf{C}_{1}$ and $\mathbf{C}_{1} \mathbf{A g}_{4}$, and by the NOE contacts attained from the NOESY spectrum (Figure S4). Hence, the 3OMeFuc4N unit was connected by an O-glycosidic linkage to position 4' of the aglycon of the next sugar unit; i.e., the polymer had a repeating unit made of an aminoacid derivative and a monosaccharide, linked together by alternating amidic and glycosidic bonds (Figure 3).

Evaluation of the absolute stereochemistry of Poly1 and of 1. The determination of the absolute configuration of $\mathbf{1}$, the monomer of Poly1, was achieved by the multistep approach summarized in Scheme 1 (full discussion in the Supporting Information), which crossed the information from the molecular modeling of two oligomers, with the experimental constraints derived from NOESY and CD spectra.

The process used to model the oligomers consisted of four sequential steps. In the first step, the aglycon was built by taking arbitrarily the combination $2 S, 3 S, 4 R$ for the three stereocenters (Figure 5), and Monte Carlo Multiple Minimum (MCMM) search returned the ${ }^{3} \mathrm{E}$ (envelope, Figure 5) conformer as the one preferred over other possible ring conformations.

In step 2, the two methyl glycosides 2 and $\mathbf{3}$ (Figure 5) were modeled by linking the aglycon to $\mathrm{D}$ - or L-configurated $3 \mathrm{OMeFuc} 4 \mathrm{~N}$ respectively. For each glycoside, the optimal orientation of the amidic linkage joining the aglycon to the sugar unit (drawn in blue, Figure 5), was evaluated by calculating the flexible map as function of the $\Phi$ and $\Psi$ dihedrals (Figure S5).

In step 3, the accumulated information enabled the construction of the dimers $\mathbf{4}$ and $\mathbf{5}$ (Figure 5) which were used to evaluate the optimal orientation of the glycosidic linkage (drawn in red in Figure 5, flexible maps in Figure S5) which connected the $30 \mathrm{MeFuc} 4 \mathrm{~N}$ unit of one monomer to the aglycon of the next unit.

Finally, the information gathered during the first three steps (Scheme 1), were used to construct two hexamers (Figure 5), Dhexa and L-hexa, which had the same oxoproline ring and differed for the absolute configuration, D or L, of 3OMeFuc4N (Figure 5).

For each hexamer, the flexibility of the glycosidic linkages was explored by Molecular Dynamic (MD) simulation (step 4, Scheme 1), an approach superior over the steps 2 or 3 because it takes into account the conformational restrictions that may arise when the monomer is part of a longer chain.

Thus, the ensemble of conformers obtained was used to calculate the interproton averaged distances, and these simulated values were compared with the experimental values (Table S4) derived from the NOESY spectrum of Poly1 (step 5 in Scheme 1). In detail, the simulated distances were calculated for the sequence $\mathrm{Ag}-3 \mathrm{OMeFuc} 4 \mathrm{~N}-\mathrm{Ag}^{\prime}$, where $\mathrm{Ag}$ indicates the aglycon linked to position 4 of the sugar moiety, while $\mathrm{Ag}^{\prime}$ is the aglycon of the next unit. Further, this evaluation focused on the sequence placed at the center of the hexamer because located in an environment similar to that of the polymer.

As result, the best agreement between the simulated and the experimental distances was found for L-hexa, which showed as discriminant interproton distances: $2.96 \AA$ between $\mathrm{H}-4$ ' of $\mathrm{Ag}$ and $\mathrm{H}-2$ of the sugar unit, and $3.34 \AA$ between $\mathrm{H}-1$ of the 3 OMeFuc4N unit and $\mathrm{H}-7^{\prime}$ (the methyl group) of $\mathrm{Ag}^{\prime}$. In the D-hexa model, these two distances were above $4.15 \AA$, hence against the experimental data; while others ( $\mathrm{H}-7^{\prime}$ of $\mathrm{Ag}^{\prime}$ with $\mathrm{H}-3$ and with $\mathrm{H}-5$ of $3 \mathrm{OMeFuc} 4 \mathrm{~N}$, Table S4) predicted by the simulation were not detected in the real sample and in the simulation of L-hexa, as well.

Hence, based on the above results, L-hexa harbored the right relative combination of all stereocenters, namely the 2 'S, 3 'S, $4^{\prime} R$ 
of the oxoproline ring and the $L$ absolute configuration of the sugar. Of note, the same agreement occurs for the mirror image of this molecule, L-hexa*, namely the hexamer having the $2^{\prime} R, 3^{\prime} R, 4^{\prime} S$ stereochemistry of the aglycon and the $\mathrm{D}$ configuration of the $30 \mathrm{MeFuc} 4 \mathrm{~N}$ unit.

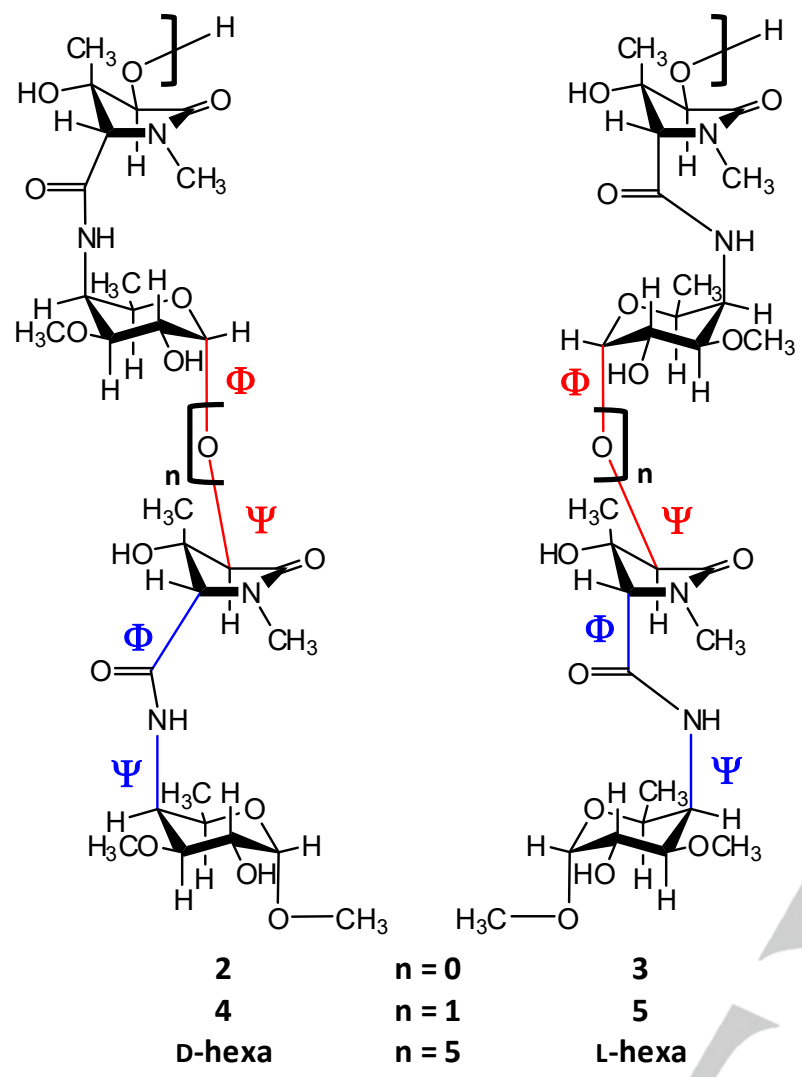

Figure 5. Structures of the molecules used to evaluate the configuration of the aglycon relatively to the $3 \mathrm{OMeFuc} 4 \mathrm{~N}$ unit. As for the aglycon, the combination $2^{\prime} S, 3^{\prime} S, 4^{\prime} R$ of the three stereocenters (see Figures 1 or 3 for numbering) was adopted, along with the ${ }^{3} \mathrm{E}$ conformation of the ring. The dihedral optimization was performed for the amide linkage (drawn in blue) of $\mathbf{2}$ and $\mathbf{3}\left(\Phi=\mathrm{H}_{2}-\mathrm{C}_{2}{ }^{-} \mathrm{C}_{1}{ }^{-}\right.$ $\mathrm{N}_{4}$ and $\Psi=\mathrm{H}_{4}-\mathrm{C}_{4}-\mathrm{N}_{4}-\mathrm{N}_{4}$ ); and for the glycosidic linkage (drawn in red) of $\mathbf{4}$ and $5\left(\mathrm{H}_{1}-\mathrm{C}_{1}-\mathrm{O}_{1}-\mathrm{C}_{4^{\prime}}\right.$, and $\left.\Psi=\mathrm{C}_{1}-\mathrm{O}_{1}-\mathrm{C}_{4^{\prime}}-\mathrm{H}_{4}\right)$. The corresponding flexible maps are reported in Figure S5.

In order to sort between L-hexa and L-hexa* the one with the same stereochemistry of Poly 1 , the $\alpha / \beta$ mixture 1 was transformed in $\mathbf{6}$, in order to measure its dichroic curve. To this end, $\mathbf{1}$ was subjected to strong alkaline hydrolysis, and the resulting methyl glycoside mixture of $30 \mathrm{MeFuc} 4 \mathrm{~N}$ devoid of the aglycon moiety, was transformed in 6 by reaction with $p$-Br-benzoyl-chloride (Figure S6). This derivative had the two chromophores installed at $\mathrm{N}-4$ and at O-2 (Figure S6), and its dichroic curve (Figure S6c) defined the absolute configuration of $30 \mathrm{MeFuc} 4 \mathrm{~N}$ as $\mathrm{D}$.

This last information (step 5, Scheme 1) allowed the selection of L-hexa* as the model responding to all the experimental data accumulated. Therefore, the monomeric unit of Poly1 consists of the aglycon $\left(2^{\prime} R, 3^{\prime} R, 4^{\prime} S\right) \quad N$-methyl-3',4'-dihydroxy-3'-methyl-5'oxoproline linked as amide to $\mathrm{N}-4$ of a 4 -amino-4-deoxy-3OMe- $\alpha$ $\mathrm{D}$-fucose as depicted in $\mathbf{1}$. In the polysaccharide, the monomeric unit 1 is linked to the next via a glycosidic linkage with the $0-4$ of the oxoproline ring of the next unit, as depicted in Figure 3.

As a general comment, it is worth noting that a 5-oxoproline residue is a rare acylating group in bacterial polysaccharides, it has been found covalently attached to the amino sugars of very few bacterial endotoxins, such as those from Vibrio cholerae 0:5, ${ }^{[10]}$ Vibrio anguillarum $\mathrm{V}-123{ }^{[11]}$ Pseudomonas fluorescens IMV 2366, ${ }^{[12]}$ Pandorea pulmonicola, ${ }^{[13]}$ Agrobacterium larrymoorei. ${ }^{[14]}$ All these 5 -oxoproline derivatives appear as $\mathrm{N}$-acyl appendages of different 6-deoxy-sugars, and none of them functions as connection for the monomeric units of the polymer, as found in this study.

Strong alkaline hydrolysis and characterization of Poly2. By taking advantage of the alkali susceptibility of the amide linkage in Poly1, the whole LPS fraction was subjected to a harsh alkaline treatment which destroyed Poly1 and returned a second polymer, Poly2 (Figure 6 and S7).

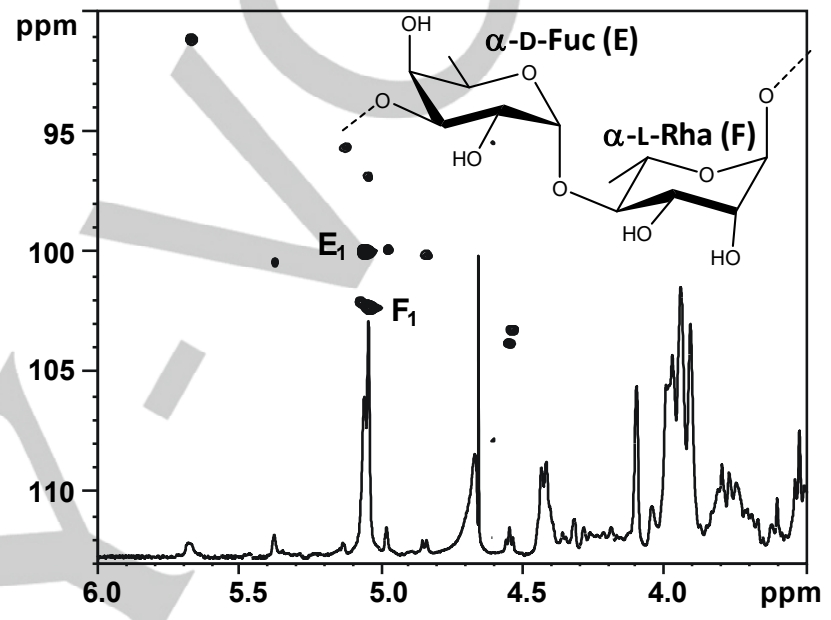

Figure 6. (500 MHZ, $313 \mathrm{k}$ ) Expansion of the anomeric region of $P$ oly2 (repeating unit structure composed of L-Rha and D-Fuc is reported in the inset). Of note, two signals are more intense than the others while the others represent the core region and the lipid A saccharide backbone of the whole LPS after full deacylation. The full spectrum is reported in Figures $\$ 7,8$.

${ }^{1} \mathrm{H}$ NMR of Poly2 (Figure 6) presented a complex array of anomeric proton signals, with two having the same intensity and more intense than all the others. These two protons almost overlapped at 5.06 and $5.04 \mathrm{ppm}$, and the combined use of the complete set of 2D NMR (Figures S7,8, Table S6) disclosed that Poly2 was a regular polymer with a disaccharide repeating unit made of 4-Rha $(\mathbf{F})$ and 3-Fuc $(\mathbf{E})$, both $\alpha$ configured at the anomeric center. Of note, the heterogeneity visible in the anomeric region of the proton spectrum was only apparent. Indeed, the alkaline treatment left attached Poly2, the OPS of the LPS, to the fragment containing the core region and the deacylated lipid $A$ moiety. Identification of this last moiety was driven by the characteristic multiplicity and chemical shift of the signal at 5.63 $\mathrm{ppm}$, that corresponds to the proton signal of the phosphorylated anomeric position of the reducing glucosamine of lipid A. ${ }^{[15]}$ Due to the low intensity of this and other signals, it was not possible to gather further information on these monosaccharide residues or about their sequence.

\section{Effect of RrTT9 LPS, Poly and Poly2 on the induction of the} plant defense system.

In line with our previous studies of the role of bacterial LPS in triggering or suppressing plant immunity, we decided to investigate the role of the O-antigens from the pathogenic Rhizobium radiobacter strain TT9. We tested the ability of the whole RrTT9 LPS, of the $\mathrm{O}$-antigen polysaccharide derived by the Smith 
degradation (Poly1) and of the polymer after strong alkaline treatment (Poly2) to activate an early innate immune response, the elicitation of the oxidative burst, in Arabidopsis thaliana, by measuring the generation of reactive oxygen species (ROS). As positive controls, we tested the purified LPS from Xanthomonas campestris pv. campestris (Xcc) and the flagellin peptide flg22. ${ }^{[12,16]}$ As a negative control, we treated the plant with water. The rationale of these experiments was to shed light on the initial stage of the interaction between Arabidopsis and the O-antigens, Poly1 and Poly2, along with the intact LPS mixture from RrTT9. None of the three tested compounds induced a defense response in Arabidopsis (Figure 7a-c), whereas $A$. thaliana responded to LPS from a plant pathogen (Xcc) and to flg22, but not to water treatment (Figure $7 \mathrm{~d}-\mathrm{f}$ ), in line with previous data. ${ }^{[17]}$

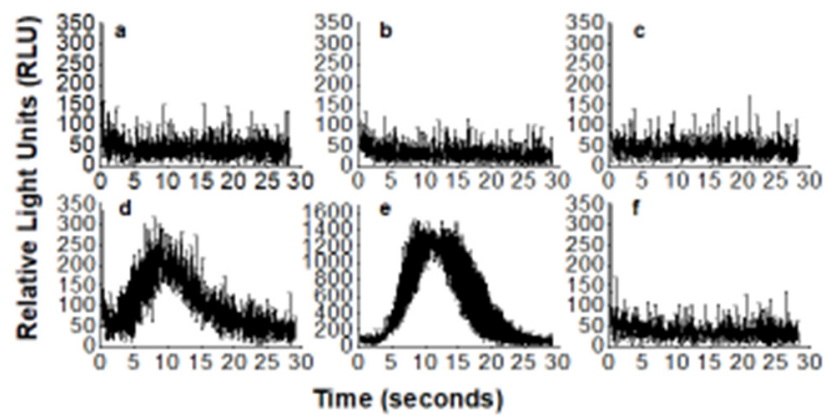

Figure 7. Elicitation of the oxidative burst in $A$. thaliana. The generation of reactive oxygen species (ROS) was measured as peroxide with a luminol assay and expressed as relative light units (RLU) in leaves of $A$. thaliana after exposure to: (a) Rhizobium radiobacter strain TT9 (RrTT9) LPS (50 $\mathrm{gg} / \mathrm{mL}$ ); (b) Poly1 (50 $\mu \mathrm{g} / \mathrm{mL})$; (c) Poly2 $(50 \mu \mathrm{g} / \mathrm{mL})$; (d) LPS from Xanthomonas campestris pv campestris $(50 \mu \mathrm{g} / \mathrm{mL})$; (e) flg22 (10 nM). Water did not induce an oxidative burst (f). Data are the means of at least four replicates. Error bars show the standard deviation.

\section{Conclusion}

The aim of this study was to evaluate the role, if any, of the coat of RrTT9 during the infection process on the plant model Arabidopsis thaliana, and to seek a possible relation between the activity measured and the structure of the LPS and/or of the OPS.

Our results show that the LPS from RrTT9 has two different Oantigens and that none of them, alone or in the LPS, triggers early defenses in Arabidopsis thaliana. This is a rather uncanonical result as LPSs of most plant pathogens do exert an immunity response from the plant. However, this finding has some analogies with the perception that plants have for symbiotic bacteria, as recently reported for another Rhizobiales, Bradyrhizobium sp. BTA1 ${ }^{[6]}$ In fact, the O-antigen of Bradyrhizobium sp. BTA1 has a peculiar structure, entirely made of a carbocyclic sugar, which we hypothesized as being the factor able to prevent the "harmful" recognition of the bacterium by its symbiotic host.

Our current results suggest that the pathogenic $R$. radiobacter TT9 develops a similar stealth strategy in order to carry on the infection process in the plant, with the advantage to prevail over other potential pathogens. An LPS coating not recognized by the host receptor(s) is indeed of great benefit to this pathogen, as its virulence will increase without the induction of the MAMP triggered immunity. RrTT9 realizes this strategy by coating itself with an LPS presenting two different $\mathrm{O}$-antigens, Poly1 and Poly2, both unique in structure.

As for Poly2, it is built up of a disaccharide repeating unit made of L-rhamnose and D-fucose (Figure 6), the latter being the less common fucose stereoisomer. Furthermore, the polysaccharide structure has not been reported so far as deduced by the query on the Bacterial Carbohydrate Structure Database repository. ${ }^{[18]}$

As for Poly1, its tricky architecture has been solved by an extensive and non-trivial integrated combination of chemical, spectroscopic and computational methods. Its monomeric unit consists of a monosaccharide, $30 \mathrm{Me}-\mathrm{D}-\mathrm{Fuc} 4 \mathrm{~N}$, and an $\alpha$-amino acid derivative, $\left(2^{\prime} R, 3^{\prime} R, 4^{\prime} S\right) N$-methyl-3',4'-dihydroxy-3'-methyl-5'oxoproline, so that the whole polymer alternates glycosidic and amidic linkages (Figure 3). Under this perspective, Poly1 is unique because it is the first polymer whose repeating unit contains a monosaccharide and an $\alpha$-aminoacid assembled together. Therefore, our view is that this O-antigen might be the first representative of a new class of natural polymers.

To the best of our knowledge, the alternance of glycosidic and amidic linkages has been reported so far only for the capsular polysaccharide from another member of the Rhizobiales, Sinorhizobium fredii $\mathrm{HH} 103$. Nevertheless, in this case there is no amino acid and the non-carbohydrate component is a hydroxybutyric amide. ${ }^{[19]}$

Furthermore, the discovery of Poly1 raises some questions: how is this polymer perceived, if at all, in the host plant? Moreover, what is its biosynthetic pathway? Finally, how many other polymers like this have gone undetected due to this structural complexity?

All in all, this is also a sensational discovery since this is the first example in which the O-antigenic part of LPS is not (strictly speaking) a real polysaccharide. Hence, the work presented here brings a new concept of polymer architecture and plant recognition of pathogenic microbes to the attention of the scientific community active in microbiology, structural biology, glycobiology and plant pathology areas.

\section{Conflict of interest}

The authors declare no conflict of interest

Keywords: Biopolymers • Structural biology • Lipopolysaccharides - Carbohydrates • Plant immunity •

[1] A. Silipo, A. Molinaro, in Endotoxins: Structure, Function and Recognition (Eds: X. Wang, P. J. Quinn), Springer, London, New York, 2010, pp. 69100.

[2] C. Alexander, E. T. Rietschel, J. Endoxin Res. 2001, 7, 167-202.

[3] A. Silipo, C. De Castro, R. Lanzetta, M. Parrilli, M. Molinaro, in Prokaryotic cell wall compounds structure and biochemistry (Eds. H. Konig, C. Herald, A. Varma), Springer-Verlag, Berlin, Germany, 2010, pp. 133-154.

[4] S. Akira, Curr. Top. Microbiol. Immunol. 2006, 311, 1-16.

[5] M. R. Leone, G. Lackner, A. Silipo, R. Lanzetta, A. Molinaro, C. Hertweck, Angew. Chem. Int. Ed. 2010, 49, 7476-7480.

[6] A. Silipo, M. R. Leone, G. Erbs, R. Lanzetta, M. Parrilli, W.-S. Chang, M. A. Newman, A. Molinaro, Angew. Chem. Int. Ed. 2011, 50, 12610-12612.

[7] E. Bedini, C. De Castro, G. Erbs, L. Mangoni, J. M. Dow, M. A. Newman, M. Parrilli, C. Unverzagt, J. Am. Chem. Soc. 2005, 127, 2414-2416.

[8] A. Silipo, G. Erbs, T. Shinya, J. M. Dow, M. Parrilli, R. Lanzetta, N. Shibuya, M. A. Newman, A. Molinaro, Glycobiol. 2010, 20, 406-419.

[9] J. M. Young, L. D. Kuykendall, E. Martínez-Romero, A. Kerr, H. Sawada Int J Syst Evol Micr 2001, 51, 89-103.

[10] K. Hermansson, P. E. Jansson, T. Holme, B. Gustavsson, Carbohydr. Res. 1993, 248, 199-211.

[11] H. Eguchi, S. Kaya, Y. Araki, Carbohydr. Res. 1992, 231, 147-158. 
[12] G. V. Zatonsky, N. A. Kocharova, S. P. Veremeychenko, E. L. Zdorovenko, A. S. Shashkov, G. M. Zdorovenko, Y. A. Knirel, Carbohydr. Res. 2002, 337, 21-23.

[13] F. Di Lorenzo, A. Silipo, A. Costello, L. Sturiale, M. Callaghan, D. Garozzo, R. Lanzetta, M. Parrilli, S. McClean, A. Molinaro, Eur. J. Org. Chem. 2012, 2243-2249.

[14] A. Molinaro, C. De Castro, R. Lanzetta, M. Parrilli, A. Raio, A. Zoina Carbohydr. Res. 2003, 338, 2721-2730.

[15] F. Di Lorenzo, A. Palmigiano, M. Pallach, D. Garozzo, M. L. Bernardini, V. Cono, M. M. Yakimov, A. Molinaro, A. Silipo, Mar Drugs. 2017, 27,15(7).
[16] C. Zipfel, S. Robatzek, L. Navarro, E. J. Oakeley, J. D. G. Jones, G. Felix, T. Boller Nature, 2004, 428, 764-767.

[17] M. A. Newman, T. Sundelin, J. T. Nielsen, G. Erbs, Front. Plant Sci. 2013, 4, 139.

[18] Ph.V. Toukach, K.S Egorova, Nucleic Acids Res. 2016, 44, D1229-D1236.

[19] A. M. Gil-Serrano, M. A. Rodriguez-Carvajal, P. Tejero-Mateo, J. L. Espartero, M. Menendez, J. Corzo, J. E. Ruiz-Sainz, A. M. BuendiaClaveria, Biochem. J. 1999, 342, 527-535. 
WILEY-VCH

\section{RESEARCH ARTICLE}

Rhizobium radiobacter strain TT9 coating is realized with an LPS mixture presenting two O-antigens, Poly1 and Poly2, both novel in structure and unable to trigger the defense system in the model plant Arabidopsis thaliana. Poly2 repeat comprises rhamnose and fucose, while Poly1 has a novel polymer architecture given by the alternance of a monosaccharide and of an aminoacid derivative.

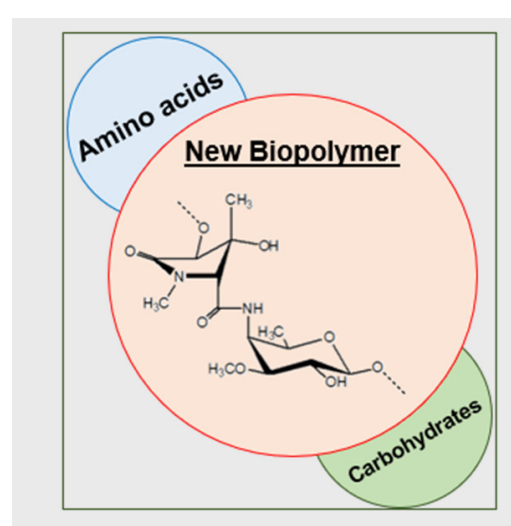

Immacolata Speciale, Flaviana Di Lorenzo, Valentina Gargiulo, Gitte Erbs, Mari-Anne Newman, Antonio Molinaro, ${ }^{*}$ Cristina De Castro*

Page No. - Page No.

A new biopolymer skeleton is produced by Rhizobium radiobacter: stoichiometric alternation of glycosydic and peptide bonds in the LPS OAntigen 